\title{
Reply to: Models for the in-host dynamics of malaria revisited: errors in some basic models lead to large over- estimates of growth rates
}

\author{
M. B. GRAVENOR* and A. L. LLOYD ${ }^{2}$ \\ ${ }^{1}$ Department of Paediatrics, University of Oxford, Institute of Molecular Medicine, Oxford OX3 9DS \\ ${ }^{2}$ Department of Zoology, University of Oxford, South Parks Road, Oxford OX1 3PS
}

(Received 24 April 1998; accepted 18 May 1998)

In the accompanying manuscript Saul (1998) points out that a model of within-host malaria population dynamics (Anderson, May \& Gupta, 1989) can exhibit unrealistically large growth rates. He suggests that this error can be avoided by replacing the parameter $r$, which is the number of merozoites produced by each parasite at schizogony, with the value $\ln (r)+1$. This substitution does not, however, address the true underlying problem with the model, namely that whilst in reality there is small variation in the distribution of Plasmodium spp. life-spans, the use of the constant rate $\alpha$ assumes an exponential, and hence much more variable, distribution. This can allow the population to increase, over the time period of the average life-span $(48 \mathrm{~h}$ in the case of Plasmodium falciparum), by factors considerably larger than $r$. Saul identifies this assumption as unrealistic in terms of the biology of malaria, but it is not remedied in the proposed model.

Within the structure of the original model there are two ways of addressing the growth rate problem. Firstly, the 'growth constant' $r$ can be replaced by the value $\ln (r)+1$, so that if all parasites reinvade, the model increases by a factor $r$ over 1 generation. As pointed out by Saul, this is an artificial device since it means that each parasite produces a reduced number of merozoites. Alternatively, a parasite can produce the observed number of merozoites, $r$, many of which do not reinvade. This is the situation in the original paper and Gravenor, McLean \& Kwiatkowski (1995). In these papers the model does grow at a reasonable rate because the parameter $\beta$ is estimated directly from observed growth rates. These points, however, are only an aside. Both the original and Saul's modified model can grow at the same rate and they both have the same distributional assumptions concerning parasite life-span.

One way to build on the original model, which was one of the first to examine within-host malaria dynamics, is to replace the term describing parasite

* Corresponding author: Department of Paediatrics, University of Oxford, Institute of Molecular Medicine, Oxford OX3 9DS. Tel: +1865 222310. Fax: +1865 222626. E-mail: michael.gravenor@bbsrc.ac.uk life-span by a term that incorporates a time delay so that every parasite has a life-span of exactly $48 \mathrm{~h}$. We suggest as an alternative, the simple addition of more age compartments to the intracellular parasite stage.

Instead of modelling the intracellular category by a single compartment, we introduce $n$ categories. As a parasite matures, it passes through the categories in sequence, and constant rates govern the transition between categories. The overall parasite life-span is now described by a sum of several exponential distributions. This distribution is considerably less variable than the single exponential previously used.

If the $n$ categories are of equal duration, we write:

$\frac{d y_{1}}{d t}=\beta x s-n \alpha y_{1}$

and

$\frac{d y_{i}}{d t}=n \alpha\left(y_{i-1}-y_{i}\right), \quad i=2,3, \ldots, n$

for intracellular parasites, and

$\frac{d s}{d t}=r n \alpha y_{n}-\delta s-\beta x s$

for merozoites. In the same way as for the basic model, it is possible to estimate $\beta$ from observed growth rates.

Note that the parameter for the reproductive event is the observed $r$ (e.g. 16) and not $\ln (r)+1$, since it is known that each parasite produces $r$ merozoites. Depending on the assumptions made about the distribution of life-spans (i.e. the number of categories), the release of $r$ merozoites per parasite can lead to the population increasing by varying factors over a 48 -h period. During the initial growth phase it can be shown that the growth factor is given by the expression $\exp \left[n\left({ }^{n} \sqrt{ } r-1\right)\right]$, which tends to $r$ as $n$ increases.

Comparing the two models we find that the original model leads to equilibrium solutions that are identical to those obtained from this expanded version. The general conclusions of Gravenor et al. (1995) then remain the same: that erythrocyte 
competition alone is insufficient to regulate parasitaemia and, in particular, that low parasitaemias can potentially account for considerably more erythrocyte destruction than previously expected (Gravenor et al. 1995, Fig. 3).

It is also important to note that the addition of age structure has the benefit of addressing key biological questions relevant to $P$. falciparum infection. We are unable to observe parasites of all ages in a malaria infection, and the biological characteristics of the parasite (such as immunogenicity and pathogenicity) can change dramatically as it matures. We have used this approach to model the age-dependent effects of fever on parasite growth (Gravenor \& Kwiatkowski, 1998) and to estimate the dynamics of mature sequestered parasites (Gravenor, Boele van Hensbroek \& Kwiatkowski, 1998).

Mathematical modelling is central to the investigation of the within-host population behaviour of malaria, about which very little is currently understood. Initial modelling will inevitably contain simplifying assumptions that can later be built upon. The use of constant rates to describe life-span is an attractive starting point, but is unrealistic in many biological systems. (Such issues have been addressed by many authors including Blythe \& Anderson (1988) in the context of epidemiology and Nowak et al. (1997) for within-host models.) It is important that the implications of these assumptions be made clear to the reader so as to aid the evaluation and development of the models.

\section{REFERENCES}

ANDERSON, R. M., MAY, R. M. \& GUPTA, s. (1989). Nonlinear phenomena in host-parasite interactions. Parasitology 99, S59-S79.

BLYTHE, s. P. \& ANDERSON, R. M. (1988). Distributed incubation and infectious periods in models of the transmission dynamics of the human immunodeficiency virus (HIV). IMA Fournal of Mathematics Applied in Medicine and Biology 5, 1-19.

GRAVENOR, M. B., MCLEAN, A. R. \& KWIATKOWSKI, D. (1995). The regulation of malaria parasitaemia: parameter estimates for a population model. Parasitology 110, 115-122.

GRAVENOR, M. B. \& KWIATKOWSKI, D. (1998). An analysis of the temperature effects of fever on the intra-host population dynamics of Plasmodium falciparum. Parasitology (in the Press).

GRAVENOR, M. B., BOELE VAN HENSBroek, M. \& KWIATKOWSKI, D. (1998). Estimating sequestered parasite population dynamics in cerebral malaria. Proceedings of the National Academy of Sciences, USA 95, 7620-7624.

NOWAK, M. A., LlOYD, A. L., VASQUEZ, G. M., Wiltrout, T. A., WAHL, L. M., BISCHOFBERGER, N., Williams, J., KINTER, A., FAUCI, A. S., HIRSCH, V. M. \& LIFSON, J. D. (1997). Viral dynamics of primary viremia and antiretroviral therapy in simian immunodeficiency virus infection. Fournal of Virology 71, 7518-7525. SAUl, A. (1998). Models for the in-host dynamics of malaria revisited: errors in some basic models lead to large over-estimates of growth rates. Parasitology 117, 403-405. 